\title{
Testing linear marginal stability in stratified shear layers
}

\author{
C. J. Howland ${ }^{1} \dagger$, J. R. Taylor ${ }^{1}$ and C. P. Caulfield ${ }^{2,1}$ \\ ${ }^{1}$ Department of Applied Mathematics and Theoretical Physics, Centre for Mathematical \\ Sciences, University of Cambridge, Wilberforce Road, Cambridge CB3 0WA, United Kingdom \\ ${ }^{2}$ BP Institute, University of Cambridge, Madingley Road, Cambridge CB3 0EZ, United \\ Kingdom
}

(Received xx; revised xx; accepted xx)

We use two-dimensional direct numerical simulations of Boussinesq stratified shear layers to investigate the influence of the minimum gradient Richardson number $R i_{m}$ on the early time-evolution of Kelvin-Helmholtz instability to its saturated 'billow' state. Even when the diffusion of the background velocity and density distributions is counter-balanced by artificial body forces to maintain the initial profiles, in the limit as $R i_{m} \rightarrow 1 / 4$ the perturbation growth rate tends to zero and the saturated perturbation energy becomes small. These results imply, at least for such canonical inflectional stratified shear flows, that 'marginally unstable' flows with $R i_{m}$ only slightly less than $1 / 4$ are highly unlikely to become 'turbulent', in the specific sense of being associated with significantly enhanced dissipation, irreversible mixing, and nontrivial modification of the background distributions without additional externally imposed forcing.

\section{Introduction}

Turbulence in the atmosphere and oceans is intermittent in time and inhomogeneous in space (Baker \& Gibson 1987). Parameterising small-scale turbulent quantities in global circulation models is thus both very challenging and very important (Ivey et al. 2008). One conventional avenue of research has been the consideration of flow instabilities, as they naturally are mechanisms by which disordered motions can arise from a laminar flow. A very commonly considered instability-mediated route to turbulence is via the so-called Kelvin-Helmholtz instability (KHI) (or perhaps more appropriately 'stratified Rayleigh instability'). This normal-mode instability develops in stably stratified shear flows with inflectional background profiles of velocity and density (or equivalently buoyancy) when the destabilising effect of the shear is sufficiently strong to overcome the stabilising effect of the stratification for infinitesimal perturbations. The finite amplitude manifestation of the KHI takes the form of elliptical vortices or 'billows', which have been observed in multiple oceanic circumstances (Smyth \& Moum 2012), including in the thermocline (Woods 1968), the abyssal ocean (van Haren \& Gostiaux 2010), and above continental shelves (Moum et al. 2003). Many laboratory experiments and numerical simulations have been performed to investigate the initiation, development and breakdown of KHI (Thorpe 1973; Fernando 1991; Klaassen \& Peltier 1985; Peltier \& Caulfield 2003), which are known to be prone, particularly for sufficiently large Reynolds numbers, to a large 'zoo' of secondary instabilities (Mashayek \& Peltier 2012a).

From consideration of the Taylor-Goldstein equation (Taylor 1931; Goldstein 1931), it is possible to derive the influential 'Miles-Howard' criterion (Miles 1961; Howard 1961),

$\dagger$ Email address for correspondence: cjh225@damtp.cam.ac.uk 
which states that a sufficient condition for linear stability of an inviscid, incompressible, stably stratified shear flow defined by a (laminar) steady parallel velocity profile $\bar{U}(z)$, and buoyancy frequency $N(z)=\sqrt{-\frac{g}{\bar{\rho}} \frac{d \bar{\rho}}{d z}}$ is that the gradient Richardson number $R i_{g}(z) \geqslant$ $1 / 4$ everywhere in the flow, where

$$
R i_{g}(z)=\frac{N^{2}}{(d \bar{U} / d z)^{2}} .
$$

Significantly, Thorpe \& Liu (2009) applied this criterion to develop the concept of 'marginal instability' to interpret and predict the existence of a range of naturally occurring stably stratified turbulent flows. In this particular context, the marginal instability of a flow is quantified by a parameter $\Phi$, which is the fractional change in flow speed required to ensure linear stability of the flow. By applying the critical value of $R i_{c}=1 / 4$ to this idea, $\Phi$ satisfies

$$
(1+\Phi)^{2}=4 R i
$$

where $R i$ is some characteristic (typically the minimum $R i_{m}$ ) value of the gradient Richardson number $R i_{g}$. A flow is then said to be marginally unstable if it is in a linearly unstable state (so $R i<1 / 4$ and hence $\Phi<0$ ) and the fractional change is small compared to unity $(|\Phi| \ll 1)$.

It is important to distinguish between this particular meaning of 'marginal stability', based around the concept of linear normal-mode instabilities growing and ultimately triggering turbulence, and an alternative meaning based around the concept of inherently nonlinear processes maintaining turbulence for sufficiently low values of a Richardson number defined in terms of the mean profiles of velocity and density. Smyth \& Moum (2013) discuss applying the 'linear' marginal instability concept as proposed by Thorpe \& Liu (2009) to turbulent flows, but also provide an alternative to the latter's linear stability arguments, to explain observations of deep cycle turbulence with a Richardson number close to $1 / 4$. They use the results of Rohr et al. (1988) which show growth and decay of stratified turbulence below and above $R i=1 / 4$ respectively, effectively thus applying the 'nonlinear' marginal stability concept to the maintenance of turbulence. In a distinct (although somewhat related) approach, Thorpe et al. (2013) add an eddy viscosity and eddy diffusivity to the Taylor-Goldstein equation to modify the critical Richardson number in the Miles-Howard criterion. These added diffusive effects model a key property of a weakly turbulent background flow, but this approach is still based around appealing to linear instability processes with (an appropriate) $R i$ close to the Miles-Howard criterion as the mechanism by which perturbations can grow to sufficiently large amplitude to maintain the turbulence. Indeed, since any small perturbation will be modified by small-scale turbulence in such a flow, the validity of performing linear stability analysis in this regime is at least formally questionable, especially if the predicted growth rate is small. Despite this, they claim that applying the concept of marginal instability to such flows may explain the behaviour of shear layers after KHI breakdown.

For inviscid flows susceptible to KHI, as $R i_{m}$ approaches $1 / 4$ from below, the exponential growth rate of the linear instabilities is predicted to drop to zero (Hazel 1972). However, this prediction does not in itself preclude the possibility that the finite amplitude 'billow' can still have significant amplitude for flows with such 'marginal' Richardson numbers, particularly in light of the results of Kaminski et al. (2017). Using a direct-adjoint-looping method, they demonstrated that billow-like structures with nontrivial amplitude can still be triggered by 'linear optimal' perturbations of small initial perturbation energy (i.e. non-normal perturbations with a structure which exhibits 
maximum transient perturbation energy growth over a finite time interval) in flows where $R i_{g}>1 / 4$ everywhere initially. However, the classical Miles-Howard criterion cited in the linear marginal stability arguments does not apply to these non-normal perturbations, and so we focus our study on normal-mode perturbations. Despite all these caveats, it is still at least conceivable that a billow could reach large amplitude at very late times for a marginally unstable flow.

Our principal aim here is to investigate whether marginal instability, in the abovediscussed sense of being based around linear stability arguments for the triggering of, rather than the maintenance of pre-existing turbulence, is useful in describing transitional flows, with finite, yet large Reynolds number $U_{0} d_{0} / \nu$, where $\nu$ is the kinematic viscosity, $d_{0}$ is the shear-layer half-depth, and $U_{0}$ is half the velocity difference across the shear layer. For simplicity, we restrict attention to flows with $\operatorname{Pr}=\nu / \kappa=1$, where $\kappa$ is the density diffusivity. We are particularly focused on determining whether saturated billows of nontrivial amplitude can develop in such flows perturbed by normal-mode perturbations associated with the classical KHI in flows where $R i_{m} \simeq 1 / 4$. Since the growth rate of such instabilities gets very small as $R i_{m}$ approaches $1 / 4$ from below, we consider three classes of flows: class ' $\mathrm{D}$ ' where the background distributions of velocity and density diffuse naturally (thus modifying and increasing $R i_{g}(z)$ with time); class 'S' with imposed body forces designed to maintain 'steady' background distributions (and hence $R i_{g}$ remains close to constant in time); and class 'A' where $U_{0}$ increases exponentially with time so that $R i_{m}$ can decrease through $1 / 4$. In particular, this last class allows us to test the viability of 'marginally unstable' flows to develop perturbation billows of significant amplitude. We are principally interested in the viability of the marginal instability/stability concepts as mechanisms to drive or trigger self-limiting turbulent flows, and so we are only interested in identifying the maximum amplitude (and the time at which this occurs) of the billows. Therefore, we restrict our numerical calculations to two dimensions, precluding any consideration of subsequent secondary instabilities, or indeed the ensuing turbulent break down and associated irreversible mixing. (As we discuss below, our observed initial maximum amplitudes of inherently two-dimensional KHI billows are consistent with the three-dimensional simulation results of Mashayek et al. (2013), giving us confidence that our two-dimensional calculations yield useful estimates for the amount of energy which can be transiently stored in a billow, and thus be ultimately available to drive turbulent motions.) The rest of the paper is organised as follows. In section 2, we describe our numerical model, the choice of domain and initial conditions. In section 3, we describe the particular characteristics of the three qualitatively different classes of flows we consider, and analyse the results of the simulations of each of these three different classes, identifying the key parameters controlling perturbation growth. Finally, we discuss the implications of our findings in section 4, particularly regarding the viability of the linear marginal (in)stability concepts as predictors of energetic turbulence and mixing in stratified shear flows.

\section{Simulation Setup}

We are interested in flows susceptible to primary instabilities of Kelvin-Helmholtz type, and so we consider dimensional (marked with an asterisk) background velocity $\bar{U}^{*}\left(z^{*}\right)$ and buoyancy $\bar{B}^{*}\left(z^{*}\right)=g^{*}\left(\rho_{a}^{*}-\bar{\rho}^{*}\right) / \rho_{a}^{*}$ distributions

$$
\bar{U}^{*}\left(z^{*}\right)=U_{0}^{*} \tanh \left(z^{*} / d_{0}\right), \quad \bar{B}^{*}\left(z^{*}\right)=\left(g^{*} \rho_{0}^{*} / \rho_{a}^{*}\right) \tanh \left(z^{*} / d_{0}^{*}\right)=B_{0}^{*} \tanh \left(z^{*} / d_{0}^{*}\right),
$$

where $g^{*}$ is the acceleration due to gravity, $\rho_{a}^{*}$ is a reference density, and the Boussinesq approximation applies so that $\rho_{0}^{*} \ll \rho_{a}^{*}$. We perform two-dimensional direct numerical 


$\begin{array}{ccccccc}\text { Group } & \text { Class } & R e & R i_{0} & N_{x} & N_{z} & E_{0} \\ \text { D1 } & \text { D: diffusive } & 1000 & 0,0.05,0.1,0.15,0.2 & 215-230 & 321 & 10^{-6} \\ \text { D2 } & \text { D: diffusive } & 2000 & 0,0.05,0.1,0.15,0.2 & 322-342 & 481 & 10^{-6} \\ \text { D3 } & \text { D: diffusive } & 4000 & 0,0.05,0.1,0.15,0.2 & 429-456 & 641 & 10^{-6} \\ \text { D4 } & \text { D: diffusive } & 6000 & 0,0.05,0.1,0.15,0.2 & 644-683 & 961 & 10^{-6} \\ \text { S } & \text { S: steady } & 4000 & 0-0.245 & 609-683 & 961 & 10^{-5} \\ \text { A } & \text { A: accelerating } & 4000 & 0.26 & 609 & 961 & 10^{-6}\end{array}$

TABle 1. Parameters for numerical simulations. The number of grid points in each direction are $N_{x}$ and $N_{z}$, which are varied with $R e$ and $R i_{0}$ to ensure accurate simulation of the flow.

simulations using the DiABLO software (Taylor 2008), which implements a combination of explicit third-order Runge-Kutta and implicit Crank-Nicholson schemes. The code solves the two-dimensional Boussinesq equations for the non-dimensional velocity, buoyancy and pressure fields $\boldsymbol{u}=(u, w), b$ and $p$ :

$$
\begin{aligned}
\nabla \cdot \boldsymbol{u} & =0 \\
\frac{\partial \boldsymbol{u}}{\partial t}+\boldsymbol{u} \cdot \nabla \boldsymbol{u} & =-\nabla p+\frac{1}{R e} \nabla^{2} \boldsymbol{u}+R i_{0} b \hat{\boldsymbol{z}} \\
\frac{\partial b}{\partial t}+\boldsymbol{u} \cdot \nabla b & =\frac{1}{\operatorname{RePr}} \nabla^{2} b
\end{aligned}
$$

Nondimensional variables are defined as $\mathbf{u}=\mathbf{u}^{*} / U_{0}^{*}, b=b^{*} / B_{0}^{*}, \mathbf{x}=\mathbf{x}^{*} / d_{0}^{*}$, and $t=$ $t^{*} /\left(d_{0}^{*} / U_{0}^{*}\right)$. The key parameters are the Reynolds number $R e=U_{0}^{*} d_{0}^{*} / \nu^{*}$, the Prandtl number $\operatorname{Pr}=\nu^{*} / \kappa^{*}$ (here $\operatorname{Pr}=1$ ), and the bulk Richardson number $R i_{0}=B_{0}^{*} d_{0}^{*} / U_{0}^{* 2}$.

The computational domain is 20 non-dimensional units in the vertical $(z)$ direction and free-slip, no flux boundary conditions are imposed at $z= \pm 10$. This prevents boundary effects from interfering with the shear layer in the centre of the domain. Periodicity is imposed in the streamwise $(x)$ direction and we choose the length of the domain to be the wavelength of the most unstable normal mode for each simulation. The initial nondimensional background profiles are hence $U(z)=B(z)=\tanh (z)$, which ensures that the gradient Richardson number

$$
R i_{g}(z)=R i_{0} \frac{d B / d z}{(d U / d z)^{2}}=R i_{0} \cosh ^{2} z \rightarrow R i_{m}=R i_{g}(0)=R i_{0},
$$

where $R i_{m}$ is the minimum, occurring at the midpoint of the shear layer $z=0$. These background profiles are perturbed by the fastest growing normal-mode perturbations $\boldsymbol{u}^{\prime}$ and $b^{\prime}$, giving the following initial conditions:

$$
\left.\boldsymbol{u}\right|_{t=0}=U(z) \hat{\boldsymbol{x}}+\epsilon \boldsymbol{u}^{\prime},\left.\quad b\right|_{t=0}=B(z)+\epsilon b^{\prime} .
$$

We calculate the normal modes using a matrix method code originally developed by Smyth \& Peltier (1990), which implements a finite $R e / P r$ generalisation of the TaylorGoldstein equation (or equivalently a stratified generalisation of the Orr-Somerfeld equation). The amplitude of the normal-mode perturbation is chosen such that an appropriate measure of the initial perturbation 'energy', defined in Kaminski et al. (2014) as

$$
E=\frac{1}{2}\left\langle\boldsymbol{u}^{\prime}, \boldsymbol{u}^{\prime}\right\rangle+\frac{R i_{0}}{2}\left\langle b^{\prime}, b^{\prime}\right\rangle=K+P,
$$

is equal to a prescribed value $E_{0}$. (As our background buoyancy distribution has nonuniform gradient, this does not correspond precisely to the sum of the perturbation kinetic 
(a)

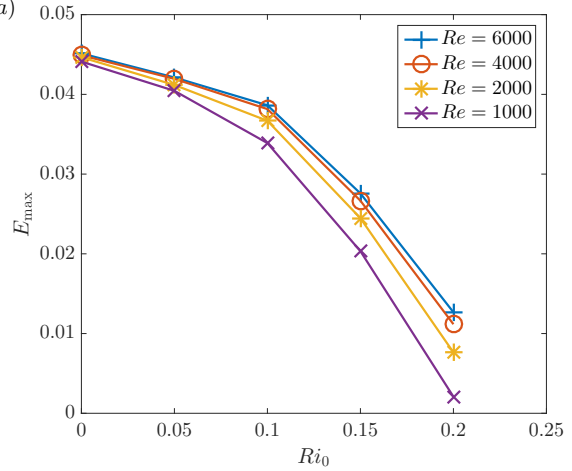

(b)

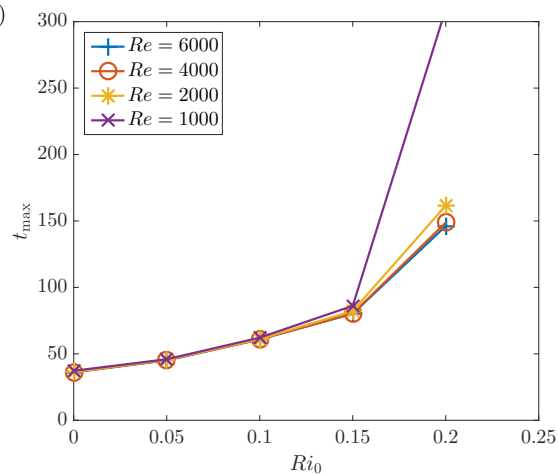

Figure 1 . For class D simulations, variation with $R e$ and $R i_{0}$ of $(a)$ peak energy $E_{\max }$ and $(b)$ time to saturation $t_{\max }$.

energy and perturbation potential energy, but we use this measure for computational convenience and consistency with the previous study of Kaminski et al. (2014).) We use the inner product $\langle\boldsymbol{u}, \boldsymbol{v}\rangle=1 / A \int_{A} \boldsymbol{u} \cdot \boldsymbol{v} \mathrm{d} x \mathrm{~d} z$, where $A$ is the area of the domain. Table 1 lists the range of parameters used.

By taking $\boldsymbol{u}^{\prime}$ and $b^{\prime}$ to be the perturbations to the initial background hyperbolic tangent profiles, we can measure the perturbation energy defined in (2.5) throughout the development of the shear layer. At early times, the perturbation energy grows exponentially as predicted by linear stability analysis. Growth of the normal mode causes redistribution of vorticity in the shear layer, which leads to a roll-up of the shear layer (Corcos \& Sherman 1976). This produces vortex-like 'core' or 'billow' regions joined by thin 'braid' structures. Fluid is entrained into the core from both sides of the shear layer, with baroclinic torques intensifying the vorticity in the braid (and elsewhere) when the flow is stratified. Soon after the roll-up, perturbation energy peaks as the primary KHI reaches its 'saturation point', which we characterise both by the maximum value of the perturbation energy, $E_{\max }$ and the time at which this occurs, $t_{\max }$.

This is the first step in the transition to turbulence of a stratified shear layer (Caulfield \& Peltier 2000). Mashayek \& Peltier (2012a) showed that a large range of secondary instabilities can develop once the saturation point has been reached, and indeed, depending on the initial perturbation structure, various merging instabilities, where one billow engulfs or drains its neighbour, can occur before saturation. Such merging events however, are suppressed at high $R e$ by the other secondary instabilities, justifying our choice of one wavelength for the length of the domain (Mashayek \& Peltier 2013). These secondary instabilities are typically three-dimensional in nature, and the subsequent energy cascade cannot be realistically modelled by two-dimensional simulations. We therefore restrict our investigation to the growth of the primary instability, and the variation in the saturation point. As noted in the introduction, we are interested in the amount of energy 'stored' in the primary billow, which would then be available to secondary instabilities and turbulent transition in a three-dimensional flow.

\section{Results}

\subsection{Class D: Diffusing shear layers}

As mentioned in the introduction, we consider three distinct classes of flow. In the first class D (simulations D1 to D4 in table 1), we consider $R e \in[1000,6000]$ and 


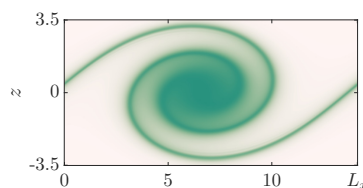

(a) $R i_{0}=0$

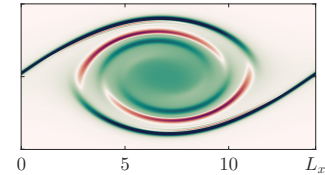

(b) $R i_{0}=0.1$

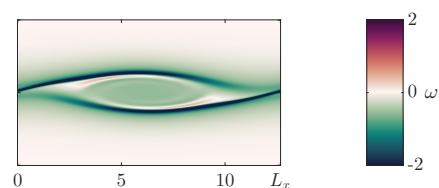

(c) $R i_{0}=0.245$

Figure 2. Snapshots of spanwise vorticity $\omega=\partial_{x} w-\partial_{z} u$ at times $t=t_{\max }$ for class $\mathrm{S}$ simulations with: (a) $R i_{0}=0$; (b) $R i_{0}=0.1$; and (c) $R i_{0}=0.245$.

$R i_{m} \in[0,0.2]$. We refer to this class as class $\mathrm{D}$ since no body forcing is added to the equations of motion and the shear layer is allowed to diffuse freely. Figure 1 shows the variation in $E_{\max }$ and $t_{\max }$ at the saturation point for these simulations. For all these simulations, we choose the initial amplitude of the perturbation to be $E_{0}=10^{-6}$ so that the perturbations exhibit an initial period of exponential growth, consistent with our linear stability calculations. At all $R e$, increasing $R i_{0}$ (and $R i_{m}$ ) produces a monotonic decrease in $E_{\max }$ as well as a monotonic increase in $t_{\max }$. The saturation point is weakly dependent on $R e$ at low values of $R i_{0}$, with a $4 \%$ decrease in $E_{\text {max }}$ between $R e=1000$ and 6000 at $R i_{0}=0.05$. The peak energy, $E_{\max }$, decreases towards zero at higher $R i_{0}$, although there is clearly nontrivial $R e$-dependence. When the perturbation amplitude is still relatively close to $E_{0}$, diffusion of the background profiles leads to an increase in $R i_{m}$ over time. The primary effect of decreasing $R e$ is to speed up this process. As a consequence, at $R i_{0}=0.2$, decreasing $R e$ monotonically decreases $E_{\max }$ and monotonically increases $t_{\max }$. Furthermore, since we define the perturbation energy as the difference from the initial velocity and buoyancy profiles defined by $U(z)=B(z)=\tanh (z)$, diffusion contributes to perturbation growth. This contribution is small compared to $E_{\max }$ except in the simulation $R e=1000, R i=0.2$, for which no clear billow structure develops. In fact, the 'saturation point' plotted in figure 1 corresponds to this simulation's end when the mean profiles have most diffused.

\subsection{Class S: Steady shear layers}

It is apparent that diffusion influences the 'saturation point' by altering the mean velocity and density profiles, particularly for $R i_{0} \simeq 1 / 4$. To investigate the behaviour at higher Richardson numbers, we introduce body forces $F_{U}$ and $F_{\rho}$ to the equations of motion to prevent diffusion of the background profiles. The governing equations become

$$
\begin{aligned}
\frac{\partial \boldsymbol{u}}{\partial t}+\boldsymbol{u} \cdot \nabla \boldsymbol{u} & =-\nabla p+\frac{1}{R e} \nabla^{2} \boldsymbol{u}+R i_{0} b \hat{\boldsymbol{z}}+F_{U} \hat{\boldsymbol{x}}, \\
\frac{\partial b}{\partial t}+\boldsymbol{u} \cdot \nabla b & =\frac{1}{\operatorname{RePr}} \nabla^{2} b+F_{\rho},
\end{aligned}
$$

where setting $F_{U}=F_{\rho}=2 \tanh (z) \operatorname{sech}^{2}(z) / R e$ ensures that the initial background profiles $\bar{U}=\bar{B}=\tanh (z)$ are steady solutions. Crucially, $R i_{m}$ of the background flow is then equal to $R i_{0}$ throughout the initial development of the primary instability. We refer to these simulations, whose properties are listed in table 1 , with $0 \leqslant R i_{0} \leqslant 0.245$ as being in class S. Since variations in $R e$ have little effect on the subsequent evolution of the flow, we fix $R e=4000$. For this class, it is natural to consider the proportion of the background kinetic energy converted to perturbation (both kinetic and potential) energy in the primary billow. At the saturation point, we therefore consider the perturbation energy density in the centre of the domain $E_{c}$ and the kinetic energy of the background 
(a)

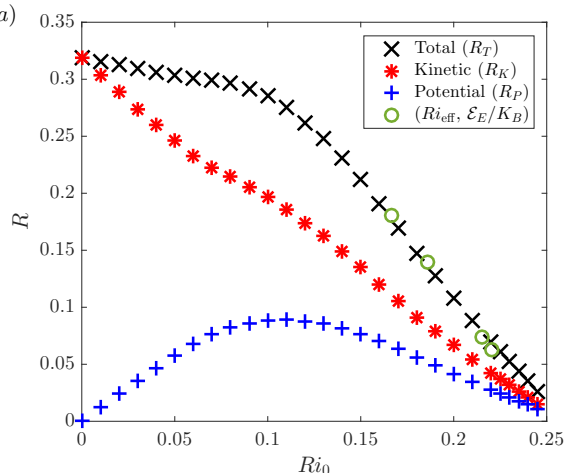

(b)

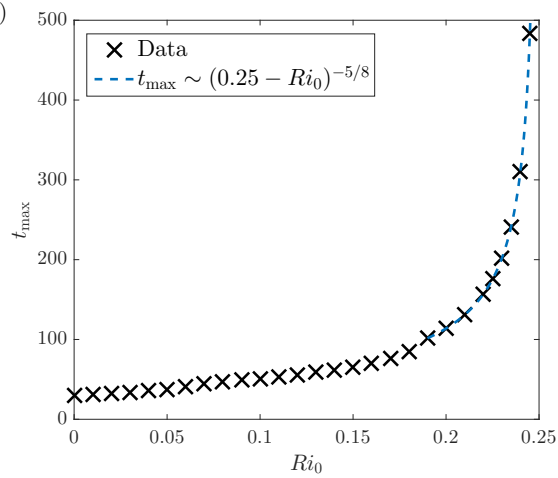

FiguRE 3. For class $\mathrm{S}$ simulations, variation with $R i_{0}$ of: (a) scaled maxima of total perturbation energy $R_{T}$ (crosses), perturbation kinetic energy $R_{K}$ (asterisks) and perturbation potential energy $R_{P}$ (pluses) as defined in (3.2); (b) $t_{\max }$. In (a) circles mark $R i_{\text {eff }}$ and the maximum value of $\mathcal{E}_{E} / K_{B}$ for class A simulations as discussed in section 3.3.

flow $K_{B}$, and calculate the ratios

$$
R_{T}=\frac{E_{c}}{K_{B}}=R_{K}+R_{P}=\frac{E_{K}+E_{P}}{K_{B}}=\frac{\frac{1}{2} \int_{-3.5}^{3.5} \int_{0}^{L_{x}} \boldsymbol{u}^{\prime} \cdot \boldsymbol{u}^{\prime}+R i_{0} b^{\prime 2} \mathrm{~d} x \mathrm{~d} z}{\frac{L_{x}}{2} \int_{-3.5}^{3.5} \tanh ^{2}(z) \mathrm{d} z},
$$

where the vertical range is chosen so that it contains all saturated billows, as is demonstrated in figure 2 for three characteristic choices of $R i_{0}=0,0.1$ and 0.245 . As is wellknown, at higher $R i_{0}$, baroclinic effects cause vorticity to become concentrated in the braid and the height of the billow to decrease (Caulfield \& Peltier 2000)

Figure 3a demonstrates a decreasing monotonic relationship between the total perturbation energy ratio $R_{T}$ at the saturation point and $R i_{0}$. The kinetic energy component $R_{K}$ decreases approximately linearly with increasing $R i_{0}$, whereas the potential energy component $R_{P}$ varies nonmonotonically, explaining the 'kink' in the variation of $R_{T}$ with $R i_{0}$ at $R i_{0} \approx 0.1$. Although we suppress diffusion of the background flows, some mixing still occurs within the billow as it rolls up, altering the mean velocity and buoyancy profiles, contributing approximately $30 \%$ of the maximum perturbation energy. Interestingly, despite the lack of linear perturbation growth at $R i_{0}=1 / 4$, the various scaled perturbation energy ratios $R_{T}, R_{K}$ and $R_{P}$ do not approach zero as $R i_{0} \rightarrow 1 / 4$. Figure $3 \mathrm{~b}$ displays the time $t_{\max }$ to the saturation point for this class, which appears to diverge as $R i_{0} \rightarrow 1 / 4$. Performing linear regression on the logarithmic values of this plot provides the divergent scaling $t_{\max } \sim\left(0.25-R i_{0}\right)^{-5 / 8}$. Crucially, it appears impossible to 'store' significant energy in a primary saturated billow from KHI when $R i_{m} \lesssim 1 / 4$, thus calling into question the applicability of the linear marginal stability concept to the triggering of turbulence in flows with $R i_{m} \simeq 1 / 4$.

\subsection{Class A: Accelerating shear layers}

Although steady flows with $R i_{m} \simeq 1 / 4$ appear not to be able to generate substantial saturated perturbation energy, to test the marginal stability/instability concepts it is also necessary to investigate whether time-dependent flows with decreasing Richardson numbers can lead to energetic billows as $R i_{m}$ drops (slightly) below $1 / 4$. To investigate this issue, we consider the final class A of simulations, which start in a linearly stable state with $R i_{0}=0.26$, and are then accelerated by body forcing which lowers $R i_{m}$ below 

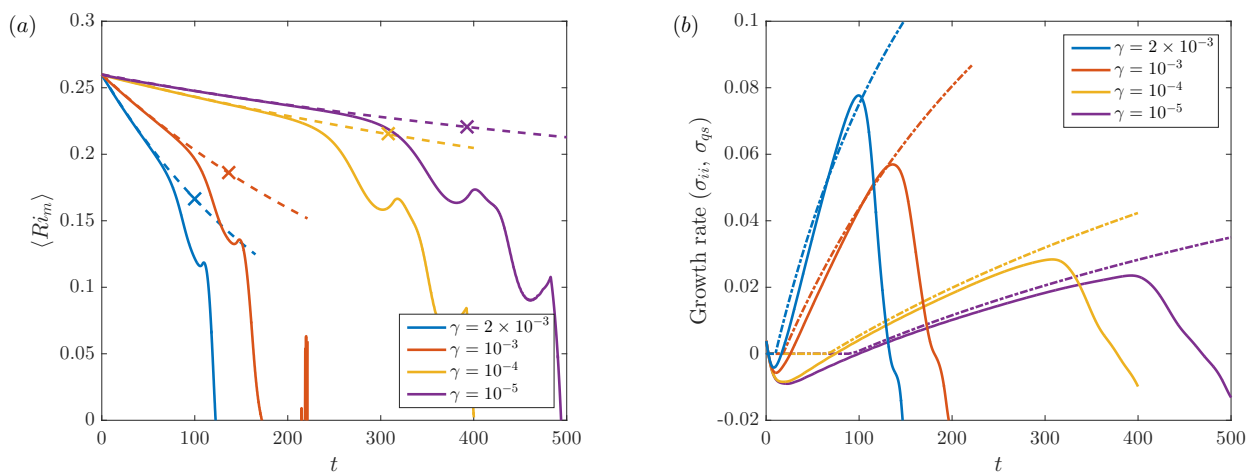

Figure 4. (a) Time series of minimum measured Richardson number $\left\langle R i_{m}\right\rangle$ from unperturbed (dashed lines) and growing (solid lines) simulations in the accelerating regime. The crosses indicate the value of $R i_{\text {eff }}$ for each simulation, at the time $t_{p}$. (b) Time series of predicted growth rate $\sigma_{q s}$ from quasi-steady linear analysis (dash-dot lines) and inferred instantaneous growth rate $\sigma_{i i}$ as defined in (3.6) (solid lines) for various forcing rates $\gamma$. The time $t_{p}$ is defined as the instant when $\sigma_{i i}$ is maximum.

1/4. Precisely, we solve (3.1) by specifying $F_{\rho}=0$ (so that buoyancy freely diffuses) and

$$
F_{U}=\left(\frac{2}{R e} \tanh (z) \operatorname{sech}^{2}(z)+\gamma \tanh (z)\right) e^{\gamma t},
$$

which thus has an accelerating background velocity solution $\bar{U}=\tanh (z) e^{\gamma t}$. Here, $\gamma$ is a forcing rate, which we prescribe as a constant between $10^{-5}$ and $2 \times 10^{-3}$. Such an accelerating background flow increases the effective Reynolds number of the flow, limiting our choice of $\gamma$ to ensure that all simulations are well-resolved up to the saturation point.

Figure $4 \mathrm{a}$ shows the effect of the forcing on the minimum mean gradient Richardson number $\left\langle R i_{m}\right\rangle$, defined by

$$
\left\langle R i_{m}\right\rangle(t)=\min _{z}\left\langle R i_{g}\right\rangle_{x}(z, t)=R i_{0} \min _{z}\left\{\frac{\langle\partial b / \partial z\rangle_{x}}{\left\langle(\partial u / \partial z)^{2}\right\rangle_{x}}\right\},
$$

where $\langle\cdot\rangle_{x}$ denotes a streamwise average. Since no growing normal-mode perturbation exists for $R i_{0}=0.26$, the initial profiles are perturbed with the most unstable mode for $R i_{0}=0.245$ and the length of the domain is set to the corresponding wavelength for that Richardson number. We also conduct unperturbed simulations for each forcing rate to track $\left\langle R i_{m}\right\rangle$ of the purely accelerating background flow in the absence of billow formation, which is plotted with dashed lines on figure 4a.

For comparison we perform a linear stability analysis for a quasi-steady background flow with $\bar{U}=\bar{B}=\tanh (z)$ and midplane Richardson number $R i_{0}=\left\langle R i_{m}\right\rangle(t)$. The analysis provides a predicted quasi-steady growth rate $\sigma_{q s}$ at each time $t$ and forcing rate $\gamma$, as shown in figure 4b. Energy is added to the system by the body forcing, so the appropriate effective perturbation energy is

$$
\mathcal{E}_{E}=E_{c}[\boldsymbol{u}, b]-E_{c}[\hat{\boldsymbol{u}}, \hat{b}],
$$

i.e. the difference between $E_{c}$ for the perturbed $(\boldsymbol{u}, b)$ and unperturbed $(\hat{\boldsymbol{u}}, \hat{b})$ simulations, where $E_{c}$ is defined in (3.2).

If the flow is linearly unstable $\boldsymbol{u}^{\prime}, b^{\prime} \sim e^{\sigma t}$, or equivalently $\mathcal{E}_{E} \sim e^{2 \sigma t}$. We can therefore test the accuracy of the linear stability analysis by calculating an inferred instantaneous 
growth rate from the perturbed nonlinear simulations

$$
\frac{1}{2 \mathcal{E}_{E}} \frac{d \mathcal{E}_{E}}{d t}=\sigma_{i i}
$$

The solid lines on figure $4 \mathrm{~b}$ show this inferred instantaneous growth rate $\sigma_{i i}$. Once the predicted quasi-steady growth rate $\sigma_{q s}$ becomes non-zero, the inferred instantaneous growth rate $\sigma_{i i}$ matches the quasi-steady linear prediction $\sigma_{q s}$ well for a certain period of time. For each choice of forcing rate $\gamma, \sigma_{i i}$ reaches a peak at some time $t_{p}$ where nonlinear effects start to become significant, before falling through zero at the saturation point, when the effective perturbation energy $\mathcal{E}_{E}$ is maximum. Interestingly, for this class of flows, the effective perturbation energy $\mathcal{E}_{E}$ at the instant when the growth rate peaks is approximately $0.75 \%$ of the initial background energy $K_{B}$, independent of the forcing rate. Simulations in class S also appear to deviate from purely 'linear' exponential-in-time perturbation growth for a similar ratio of perturbation energy to background energy $K_{B}$. The remaining perturbation growth depends on the effective Richardson number $R i_{\text {eff }}$, i.e. the value of $\left\langle R i_{m}\right\rangle$ in the unperturbed flow at the time instant $t_{p}$ when $\sigma_{i i}$ reaches its peak value in the (perturbed) simulations, marked with crosses in figure 4a. The maximum value of the appropriately scaled perturbation energy $\mathcal{E}_{E} / K_{B}$ at the saturation points is plotted (with circles) against $R i_{\text {eff }}$ on figure $3 \mathrm{a}$, showing striking agreement with the simulations in the steady class $\mathrm{S}$. It appears that the maximum amplitude of the nonlinear billow state is controlled by the minimum value of $R i_{g}$ of the background flow relatively early in the development of the instability (specifically when nonlinear effects start to reduce the inferred instantaneous growth rate), and that such maximum amplitude is very small when the relevant $R i_{0} \simeq 1 / 4$, even in accelerating flows.

\section{Discussion and Conclusions}

We have performed three classes of two-dimensional direct numerical simulations of stratified shear layers susceptible to KHI: class D where the background flow profiles are free to diffuse; class $\mathrm{S}$ where body forcings allow the initial background flow profiles to be steady solutions of the governing equations; and class A where the background velocity profile accelerates. In each class, the saturated amplitude of the nonlinear billow state decreases as the initial minimum gradient Richardson number, $R i_{m}$, increases. In class $\mathrm{D}$, $R e$ becomes important as $R i_{m} \rightarrow 1 / 4$ since diffusion of the background flow increases the Richardson number to a linearly stable value. For class $\mathrm{S}$, where body forcing keeps $R i_{m}$ constant, a clear monotonic relationship arises between $R i_{m}$ and the saturated amplitude of the nonlinear state. The time at which this saturated amplitude occurs diverges as $R i_{m} \rightarrow 1 / 4$, while the saturated amplitude of the billow decreases to a small but nonzero value. This limit yields flattened billows, as shown in figure 2c. It is important to appreciate that these results are not direct consequences of the Miles-Howard criterion, which only considers the exponential growth of infinitesimal perturbations on steady, inviscid flows, and not the properties of potentially ensuing nonlinear states.

We find that the instabilities which develop in the accelerating shear layers of class $\mathrm{A}$ are still, at early times, well-described by quasi-steady linear stability analyses using an instantaneous Richardson number. The saturated energy of these billows is still consistent with the relationship shown in figure $3 \mathrm{a}$, reaffirming that the saturated amplitude is restricted by $R i_{m}$. We find that nonlinear effects become important when a perturbation reaches an amplitude corresponding to $0.75 \%$ of the initial background kinetic energy.

We conclude that these results are not consistent with the connection between marginally unstable flow states and the generation or 'triggering' of turbulence through 
unstable linear normal-mode disturbances. In particular, the application of the MilesHoward criterion to provide a stability boundary for naturally occurring turbulent shear flows appears inconsistent with the demonstrated nonlinear development of KHI in laminar states at high $R e$. Indeed, our results are broadly consistent with studies of internal solitary waves, which have similarly suggested that wave breaking requires the minimum Richardson number in the pycnocline to be below approximately 0.1 (Lamb 2014). We hypothesise that vigorous energy injection to finite amplitude structures which have the potential to trigger turbulent transition requires minimum Richardson numbers within the flow to be substantially below $1 / 4$.

This hypothesis is not inconsistent with the observations discussed by Thorpe \& Liu (2009) and Smyth \& Moum (2013), since we are concerned with the laminar states required for turbulent transition rather than the ensuing turbulent flow itself. However, the use of linear stability analysis by Thorpe \& Liu (2009) and Thorpe et al. (2013) to determine the stability of turbulent shear flows by the Miles-Howard criterion does not appear valid given our results. Since the nonlinear structures that develop in our marginally unstable laminar flows are very small and take a long time to saturate, any linear perturbation in a similarly marginal turbulent flow is likely to be strongly influenced by the surrounding turbulence. This introduces uncertainty to both the development of a linear perturbation and the nature of its finite amplitude manifestation as a nonlinear billow structure. We conclude that the application of marginal stability to naturally occurring turbulent flows should be based on results concerning the growth and decay of stratified turbulence around certain Richardson numbers (e.g. Rohr et al. (1988), Zhou et al. (2017)), rather than linear stability arguments.

Although we have only performed simulations in a two-dimensional domain, we remain confident that our results have relevance to fully three-dimensional realisations of these flows. By computing the turbulent kinetic energy of the class S simulations, we obtain comparable values at saturation to those found in figure $3 \mathrm{~b}$ of the three-dimensional study of Mashayek et al. (2013). The peak energy we get takes slightly higher values than Mashayek et al. (2013) because of the reduced diffusion keeping the minimum Richardson number at a lower value in our case. For our highest $R i_{0}$ values no three-dimensional studies have been performed, but Mashayek \& Peltier (2012b) found that the growth rates of all secondary instabilities decrease as $R i_{0}$ is increased past 0.12 . We therefore believe that the behaviour of the two-dimensional roll-up is still important at higher $R i_{0}$.

It is important to remember that we have only considered the nonlinear development of a linear normal-mode perturbation. As noted in the introduction, Kaminski et al. (2017) have shown that sufficiently large amplitude perturbations with the structure of a linear optimal perturbation can still develop into a 'KH-like' billow state for flows with $R i_{m}$ as high as 0.4 , which may perhaps explain why the maximum energy in figure 3a does not tend to zero as $R i_{m} \rightarrow 1 / 4$. Indeed, with a specific type of large forcing, a saturated nonlinear overturning billow structure may possibly develop at $R i_{m}=1 / 4$ through a different (and inherently transient, non-normal) mechanism, distinct from perturbation by a classical normal-mode linear instability.

Although flows in the atmosphere or oceans are far more complex than those considered here, our results from time-dependent shear layers (class A) suggest that minimum values of $R i_{g}$ directly control the saturated amplitude of Kelvin-Helmholtz billows. In particular, we have shown that the time for the perturbation energy to saturate becomes very long as $R i_{m} \rightarrow 1 / 4$ and the billow amplitude is small in this limit, implying that a laminar flow in this state is unlikely to become turbulent. For example, using the typical value $1 \mathrm{~m}$ for a shear layer half-depth, and velocity difference $2 U_{0}^{*}=7.5 \times 10^{-2} \mathrm{~ms}^{-1}$ from measurements by van Haren \& Gostiaux (2010) to calculate an advective time scale 
gives $t_{\max }$ greater than 22 minutes for $R i_{0} \geqslant 0.2$. At $R i_{0}=0.24$, the appropriately scaled saturation time becomes longer than an hour. Further work to examine the effect of ambient turbulence or internal waves on the saturated billow amplitudes would be invaluable for quantifying the potential for enhanced mixing and turbulence of sheardriven overturning structures in the ocean.

\section{Acknowledgements}

We thank three anonymous referees whose thoughtful and constructive comments have substantially improved this paper. C.J.H. is supported by the Cambridge Earth System Science NERC DTP [NE/L002507/1]. The research activity of J.R.T. and C.P.C. is supported by EPSRC Programme Grant EP/K034529/1 entitled 'Mathematical Underpinnings of Stratified Turbulence'. Valuable discussions with the rest of the MUST team are gratefully acknowledged. The data used to create figures 1,3 and 4, and the initial condition header data for DIABLO for the simulations outlined in table 1 are available at https://doi.org/10.17863/CAM.17349.

\section{REFERENCES}

Baker, Mark A. \& Gibson, Carl H. 1987 Sampling Turbulence in the Stratified Ocean: Statistical Consequences of Strong Intermittency. Journal of Physical Oceanography 17 (10), 1817-1836.

Caulfield, C. P. \& Peltier, W. R. 2000 The anatomy of the mixing transition in homogeneous and stratified free shear layers. Journal of Fluid Mechanics 413, 1-47.

Corcos, G. M. \& Sherman, F. S. 1976 Vorticity concentration and the dynamics of unstable free shear layers. Journal of Fluid Mechanics 73 (2), 241-264.

Fernando, H J S 1991 Turbulent Mixing in Stratified Fluids. Annual Review of Fluid Mechanics 23, 455-493.

Goldstein, S. 1931 On the Stability of Superposed Streams of Fluids of Different Densities. Proceedings of the Royal Society A: Mathematical, Physical and Engineering Sciences 132 (820), 524-548.

van Haren, Hans \& Gostiaux, Louis 2010 A deep-ocean Kelvin-Helmholtz billow train. Geophysical Research Letters 37 (3), 1-5.

HAZEL, P 1972 Numerical studies of the stability of inviscid parallel shear flows. Journal of Fluid Mechanics 51 (1), 39-61.

Howard, L. N. 1961 Note on a paper of John W. Miles. Journal of Fluid Mechanics 10 (4), $509-512$.

Ivey, G. N., Winters, K. B. \& Koseff, J. R. 2008 Density Stratification, Turbulence, but How Much Mixing? Annual Review of Fluid Mechanics 40, 169-184.

Kaminski, A. K., Caulfield, C. P. \& Taylor, J. R. 2014 Transient growth in strongly stratified shear layers. Journal of Fluid Mechanics 758 (R4), 1-12.

Kaminski, A. K., Caulfield, C. P. \& Taylor, J. R. 2017 Nonlinear evolution of linear optimal perturbations of strongly stratified shear layers. Journal of Fluid Mechanics 825, 213-244.

Klatassen, G. P. \& Peltier, W. R. 1985 Evolution of Finite Amplitude KelvinHelmholtz Billows in Two Spatial Dimensions. Journal of the Atmospheric Sciences 42 (12), 13211339 .

Lamb, Kevin G. 2014 Internal Wave Breaking and Dissipation Mechanisms on the Continental Slope/Shelf. Annual Review of Fluid Mechanics 46, 231-254.

Mashayek, A., Caulfield, C. P. \& Peltier, W. R. 2013 Time-dependent, non-monotonic mixing in stratified turbulent shear flows: implications for oceanographic estimates of buoyancy flux. Journal of Fluid Mechanics 736 (2013), 570-593.

Mashayek, A. \& Peltier, W. R. $2012 a$ The zoo of secondary instabilities precursory to stratified shear flow transition. Part 1 Shear aligned convection, pairing, and braid instabilities. Journal of Fluid Mechanics 708 (2012), 5-44. 
Mashayek, A. \& Peltier, W. R. $2012 b$ The zoo of secondary instabilities precursory to stratified shear flow transition. Part 2 The influence of stratification. Journal of Fluid Mechanics 708 (2012), 45-70.

Mashayek, A. \& Peltier, W. R. 2013 Shear-induced mixing in geophysical flows: does the route to turbulence matter to its efficiency? Journal of Fluid Mechanics $\mathbf{7 2 5}$ (2013), 216261.

MiLes, John W. 1961 On the stability of heterogeneous shear flows. Journal of Fluid Mechanics 10 (4), 496-508.

Moum, J. N., Farmer, D. M., Smyth, W. D., Armi, L. \& Vagle, S. 2003 Structure and Generation of Turbulence at Interfaces Strained by Internal Solitary Waves Propagating Shoreward over the Continental Shelf. Journal of Physical Oceanography 33 (10), 20932112.

Peltier, W. R. \& Caulfield, C. P. 2003 Mixing Efficiency in Stratified Shear Flows. Annual Review of Fluid Mechanics 35, 135-167.

Rohr, J. J., Itsweire, E C, Helland, K. N. \& Atta, C. W. Van 1988 Growth and decay of turbulence in a stably stratified shear flow. Journal of Fluid Mechanics 195, 77-111.

Smyth, William D. \& Moum, James N. 2012 Ocean mixing by Kelvin-Helmholtz instability. Oceanography 25 (2), 140-149.

Sмyтн, W. D. \& Moum, J. N. 2013 Marginal instability and deep cycle turbulence in the eastern equatorial Pacific Ocean. Geophysical Research Letters 40 (23), 6181-6185.

Smyth, W. D. \& Peltier, W. R. 1990 Three-dimensional primary instabilities of a stratified, dissipative, parallel flow. Geophysical \& Astrophysical Fluid Dynamics 52 (4), 249-261.

TAYLOR, G. I. 1931 Effect of Variation in Density on the Stability of Superposed Streams of Fluid. Proceedings of the Royal Society A: Mathematical, Physical and Engineering Sciences 132 (820), 499-523.

TAYLOR, John R. 2008 Numerical simulations of the stratified oceanic bottom layer. PhD thesis, University of California, San Diego.

Thorpe, S. A. 1973 Experiments on instability and turbulence in a stratified shear flow. Journal of Fluid Mechanics 61 (4), 731-751.

Thorpe, S. A. \& Liu, Zhiуu 2009 Marginal Instability? Journal of Physical Oceanography 39 (9), 2373-2381.

Thorpe, S. A., Smyth, W. D. \& Li, Lin 2013 The effect of small viscosity and diffusivity on the marginal stability of stably stratified shear flows. Journal of Fluid Mechanics 731, 461-476.

Woods, J. D. 1968 Wave-induced shear instability in the summer thermocline. Journal of Fluid Mechanics 32 (4), 791-800.

Zhou, Qi, Taylor, John R. \& Caulfield, C. P. 2017 Self-similar mixing in stratified plane Couette flow for varying Prandtl number. Journal of Fluid Mechanics 820 (May), 86-120. 